Ophthalmologe 2009 · 106:97-98 DOI 10.1007/s00347-008-1862-4

Online publiziert: 22. Januar 2009

๑) Springer Medizin Verlag 2009

\author{
B. Wissinger \\ Molekulargenetisches Labor, Forschungsinstitut für Augenheilkunde, \\ Department für Augenheilkunde, Universitätsklinikum Tübingen
}

\title{
Zapfendystrophien - von der Bedeutsamkeit einer Minderheit von Photorezeptoren
}

Die menschliche Netzhaut ist mit ca. 125 Mio Photorezeptoren ausgestattet; nur etwa 5\% davon sind Zapfen. Dennoch sind die Zapfen von elementarer Bedeutung für die Leistungsfähigkeit unseres visuellen Systems. Diese besondere Leistungsfähigkeit des Zapfenphotorezeptorsystems spiegelt sich im Wesentlichen in vier Funktionsparametern unseres Sehsystems wieder:

- räumliches Auflösungsvermögen,

- zeitliches Auflösungsvermögen,

- lichtintensitätsabhängiges Adaptationsvermögen und

- Farbensehen.

In der Fovea finden sich ausschließlich Zapfen, die durch ihre hohe Packungsdichte von 140.000 Zapfen pro $\mathrm{mm}^{2}$ und ihre 1:1-Verschaltung auf Bipolar- und Ganglienzellen unser räumliches Auflösungsvermögen von $0,5-1^{\circ}$ Winkelgrad primär bestimmen. Anders als die Stäbchen, die auf die Detektion von Lichtreizen geringer Lichtintensität spezialisiert sind und als Anpassung darauf die Erregung auf rezeptoraler und postrezeptoraler Ebene integrieren und summieren, erlaubt der Arbeitsbereich der Zapfen bei mesopischen und photopischen Lichtintensitäten eine erhöhte zeitliche Auflösung der Reize. In Abhängigkeit von der Lichtintensität kann unser Zapfensystem eine Reizfrequenz von bis $\mathrm{zu} 6 \mathrm{o} \mathrm{Hz}$ auflösen. Eine besonders bemerkenswerte Leistung des Zapfensystems ist die Adaptation an Licht unterschiedlicher Intensität. Man denke an die Unterschiede der Leuchtdichten zwischen einem spärlich beleuchteten Raum und der im Freien an einem Sommertag. Verschiedene bioche- mische und neuronale Mechanismen erlauben unserem Zapfensystem eine Anpassung an Lichtintensitäten, die um 7-9 log-Stufen differieren. Die Präsenz dreier Subtypen von Zapfen (Blau-, Grün- und Rotzapfen) mit unterschiedlicher spektraler Empfindlichkeit erschließt uns schließlich einen deutlich verbreiterten Bereich elektromagnetischer Strahlung von 400-760 nm und vermittelt uns eine zusätzliche visuelle Qualität, die Farbwahrnehmung und Farbdifferenzierung.

Man kann daher mit gutem Grund das Zapfenphotorezeptorsystem als „Krönung des menschlichen Sehsystems" bezeichnen.

Entsprechend gravierende Einschränkungen, was die Leistungsfähigkeit unseres visuellen Systems betrifft, werden durch Erkrankungen verursacht, die das dauerhafte Überleben und die Funktion der Zapfen betreffen. Diese Ausgabe von "Der Ophthalmologe“ widmet sich mit zwei Übersichtsartikeln der Kerngruppe solcher Erkrankungen, den Zapfendystrophien und Zapfendysfunktionen, deren Ursache primär in Defekten der Zapfen selbst begründet ist. Ein wesentliches Kennzeichen der Zapfendystrophien und Zapfendysfunktionen ist die Heterogenität in ihrem klinischen Erscheinungsbild. Der Beitrag von U.Kellner und S. Kellner [1] gibt aus dem Blickwinkel ihrer langjährigen praktischen Erfahrung auf dem Gebiet der Netzhautdystrophien eine Übersicht über das Spektrum und die klinischen Merkmale und Charakteristika der Zapfendystrophien. Mit der Darstellung des Spektrums und der Aussagekraft klinischer Diagnoseverfahren sowie der Darlegung differenzialdiagnostischer
Probleme zielt dieser Artikel neben dem wissenschaftlich interessierten Ophthalmologen insbesondere auch auf die Anwendung in der klinische Praxis und die Wissensvermittlung an junge Assistenzund Fachärzte ab.

Zapfendystrophien und Zapfendysfunktionen sind v. a. hereditärer Natur und die genetische Ursachenforschung dieser Erkrankungen hat in den letzten Jahren beeindruckende Fortschritte erziehlt. Der Beitrag von S. Kohl [2] gibt dazu eine aktuelle Übersicht und vermittelt einen Eindruck von der Komplexität und Heterogenität der beteiligten Gene und Schadensmechanismen. Die Beschäftigung und Auseinandersetzung mit der genetischen Thematik ist dabei nicht Selbstzweck. Man muss kein Prophet sein, um zu prognostizieren, dass in naher Zukunft genetische Daten zur Ursache oder Veranlagung für eine Erkrankung sowie zur Verträglichkeit und Effektivität von Pharmaka das diagnostische Spektrum in vielen Bereichen ergänzen und erweitern werden und das ärztliche Handeln maßgeblich beeinflussen. Einen ersten Blick auf dieses neue Zeitalters gibt der am Institut für Humangenetik der Universität Regensburg sich in Entwicklung befindliche Diagnostik-Chip (RetChip), mit dem in einem Analyselauf die Gensequenz von 72 Genen für erbliche Netzhauterkrankungen ermittelt wird. Auf der Basis der rasanten Weiterentwicklung der Sequenziertechnologie erwartet man sogar, dass bereits bis zum Jahr 2015 das Gesamtgenom eines Individuums für 1000 US-Dollar ermittelt werden kann. Die Bewertung dieser Daten ist jedoch ein bislang noch nicht gelöstes Problem. 
Erkrankungen der Zapfenphotorezeptoren stellen sich bezüglich der visueller Funktionsparameter und des Fundusbefunds sowie des Erkrankungsbeginns und des Verlaufs als eine sehr heterogene Gruppe dar. Die Auftrennung in reine Zapfendystrophien und ZapfenStäbchen-Dystrophien oder die Unterscheidung von progressiven und stationären Formen mag für die Beschreibung des Ist-Zustands des einzelnen Patienten sinnvoll sein. Für die Gesamtgruppe ist sie jedoch eher willkürlich, da sie sich als kontinuierliches Spektrum von Subtypen und Verlaufsformen darstellt. Auch ätiologisch sind solche Differenzierungen nicht haltbar. So findet man beispielsweise bei mehreren Betroffenen innerhalb einer Familie häufig ausgeprägte Unterschiede in der klinischen Ausprägung und im Verlauf. Dies kann im Fall von Mutationen im $\mathrm{PRPH}_{2} / \mathrm{RDS}-\mathrm{Gen}$ soweit gehen, dass Mitglieder einer Familie sich mit differenten klinischen Krankheitsbildern präsentieren [3]. In anderen Fällen determiniert die Art und/oder Lage der Mutation oder die Dominanz bzw. Rezessivität der Mutation die Art und Schwere der klinischen Ausprägung.

In der Mehrzahl der bekannten Fälle ist der molekulare Defekt in Ausfällen oder Fehlfunktionen von Komponenten der Phototransduktionskaskade oder Strukturproteinen des Photorezeptors begründet. Im Vergleich zu den Stäbchenphotorezeptoren sind die Biochemie und die Physiologie der Zapfen noch immer wenig erforscht, und damit ist auch die Kenntnis der beteiligten Faktoren weitaus geringer. Hier wird der geringe Anteil der Zapfen in der Netzhaut der üblichen Studienobjekte zum entscheidenden Nachteil. Es ist daher zu erwarten, dass die Liste der für Zapfendystrophien und Zapfenfunktionsstörungen ursächlichen Gene noch längst nicht komplett ist.

Für die Analyse der zellulären Pathophysiologie von Netzhautdystrophien ist in den letzten Jahren eine Reihe von Modellsystemen in verschiedenen Spezies (insbesondere Hund, Maus, Ratte, Zebrafisch) identifiziert bzw. entwickelt worden. Durch die heutzutage routinemäßig anwendbaren Methoden der gezielten genetischen Modifikation hat die Maus als Studienobjekt für die Erforschung here- ditärer Netzhauterkrankungen weite Verbreitung gefunden. So stehen bereits für etwa die Hälfte aller bekannten Gene für Zapfendystrophien und Zapfenfunktionsstörungen Mausmutanten zur Verfügung. Mit solchen Modellen können sowohl die prinzipiellen Mechanismen der Degeneration als auch die durch die genetische Veränderung spezifisch verursachten Störungen der Zellphysiologie studiert werden. Aufgrund der geringen Zahl und Größe der Zapfen sowie der Unterschiede in der Neurophysiologie des Zapfensystems zwischen Maus und Mensch ist es jedoch dringend erforderlich, gerade auch für Therapiestudien geeignetere Modellsysteme heranzuziehen. Interessanterweise gibt es eine größere Zahl von natürlichen Hundemutanten für Netzhautdystrophien, die in den letzten Jahren identifiziert werden konnten und einen wichtigen Zwischenschritt in der Therapieentwicklung darstellen [4].

Zapfendystrophien und Zapfenfunktionsstörungen sind sehr seltene Erkrankungen (Prävalenz ca. 1:20.00o), sodass der niedergelassene Augenarzt nur selten damit konfrontiert wird. Wichtig sind dabei die Erkennung einer Netzhautdystrophie und die Überweisung an einen Spezialisten oder eine Universitätsklinik mit entsprechenden Diagnose- und Versorgungsstrukturen für diese Patienten. Derzeit gibt es keine kurativen Behandlungsmöglichkeiten für Patienten mit erblichen Netzhauterkrankungen. Umso wichtiger sind eine optimale Versorgung der Patienten mit geeigneten Sehhilfen und eine sorgsame Sozialberatung. Gerade in einem operativen und therapieorientierten Fach wie der Augenheilkunde nehmen Patienten mit erblichen Netzhauterkrankungen deshalb eine besondere Stellung ein, die besondere Anstrengungen in der Ursachen- und Therapieforschung erfordert. Hoffnung machen hier erste erfolgreiche gentherapeutische Versuche bei Patienten mit Mutationen im RPE65-Gen und Phase-I-Studien zur Implantation von Sehprothesen [5, 6, 7]. Noch in der frühen Forschungs- bzw. präklinischen Entwicklungsphase befinden sich Ansätze zur Transplantation von Stamm- oder Vorläuferzellen bzw. die Anwendung neuroprotektiver Faktoren. Die Erwartung in diese Ansätze ist hoch und die Hoffnung groß, dass in nicht allzu ferner Zukunft Patienten mit Zapfendystrophien und Zapfenfunktionsstörungen die „Krönung unseres Sehsystems“ erhalten oder wiedergegeben werden kann.

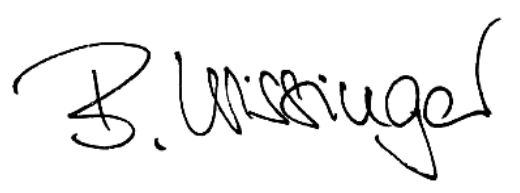

B. Wissinger

\section{Korrespondenzadresse \\ Prof. Dr. B. Wissinger}

Molekulargenetisches Labor, Forschungsinstitut für Augenheilkunde, Department für Augenheilkunde, Universitätsklinikum Tübingen Röntgenweg 11, 72076 Tübingen wissinger@uni-tuebingen.de

Interessenkonflikt. Der korrespondierende Autor gibt an, dass kein Interessenkonflikt besteht.

\section{Literatur}

1. Kellner U, Kellner S (2009) Klinik und Diagnostik der Zapfendystrophien. Ophthalmologe 106:99108

2. Kohl S (2009) Genetische Ursachen von Erkrankungen der retinalen Zapfen-Photorezeptoren. Ophthalmologe 106:109-115

3. Boon CJ, den Hollander Al, Hoyng CB et al (2008) The spectrum of retinal dystrophies caused by mutations in the peripherin/RDS gene. Prog Retin Eye Res 27(2):213-235

4. Petersen-Jones S (2005) Advances in the molecular understanding of canine retinal diseases. J Small Anim Pract 46:371-380

5. Bainbridge JW, Smith AJ, Barker SS, Robbie S et al (2008) Effect of gene therapy on visual function in Leber's congenital amaurosis. N Engl J Med 358:2231-2239

6. Maguire AM, Simonelli F, Pierce EA, Pugh EN Jr et al (2008) Safety and efficacy of gene transfer for Leber's congenital amaurosis. N Engl J Med 358:2240-2248

7. Gekeler F, Zrenner E (2005) Stand des subretinalen Implantatprojekts. Eine Übersicht. Ophthalmologe 102:941-949 